
\title{
25 Research Soure \\ Novel methodology to predict hypoglycaemia rates with basal insulin in real-world populations
}

\section{Zsolt Bosnyak \\ Fang Liz Zhou \\ Javier Jimenez \\ Rachele Berria}

\section{Video Abstract}

Keywords: hypoglycemia, basal insulin, insulin, glucose, second-generation basal insulin, glargine, degludec, hemoglobin, detemir, randomized clinical trial, RCT, machine learning, LIGHTNING, modeling, medical record, prediction, clinical biology, Diabetes Therapy, Sanofi, diabetes

Posted Date: September 23rd, 2019

DOI: https://doi.org/10.21203/rs.2.14984/v2

License: @ (i) This work is licensed under a Creative Commons Attribution 4.0 International License. Read Full License 


\section{Abstract}

People with diabetes who require basal insulin to achieve blood glucose control can be at risk of hypoglycaemia, where blood glucose levels drop too low. In randomised clinical trials (or RCTs), use of second-generation basal insulin analogues, such as insulin glargine 300 units/mL (known as glargine 300 ) and insulin degludec, results in similar glycated haemoglobin reductions compared with firstgeneration basal insulin analogues, such as glargine 100 and insulin detemir, but with less hypoglycaemia. However, it is not known whether these results translate directly to routine clinical practice, as RCTs often apply strict inclusion and exclusion criteria, meaning that they may not be generalisable to real-life situations. Electronic medical records are a source of rich real-world data, but using them to make comparisons between different treatments can be difficult because results might be biased by confounding data, something that the randomisation in RCTs is designed to minimise. In order to make the most of large amounts of data, such as those from electronic medical records, computers can be programmed to model complex data relationships and can even 'learn' from the data to make predictions. This is a process called 'machine learning'. The LIGHTNING study uses advanced methods, including machine learning, to predict hypoglycaemia rates in people with type 2 diabetes using first- and second-generation basal insulin analogues, by analysing electronic medical records. Factors that contribute to hypoglycaemia rates in patients using a particular basal insulin are first modelled using part of the dataset called the training dataset. This basal-insulin-specific model is then applied to the rest of the dataset (called the 'test dataset') to see if it accurately predicts hypoglycaemia rates in this subset. Changes are made to the model to improve prediction accuracy, before the model is applied to the test dataset again, then changed again, then tested again, and so on, until prediction is optimized. At that point, the optimized model is applied to the full dataset, irrespective of what treatment the patients were using, to give a prediction of what hypoglycaemia rates would be if all patients were using the basal insulin being modelled. This whole process is repeated many times for a particular basal insulin to obtain estimates of variability), and each basal-insulin-specific hypoglycaemia prediction model is generated in the same way. Real-world data are an important adjunct to RCTs, and it is hoped that the predictive models generated from the LIGHTNING study will aid clinical decision making. 